\title{
Effect of a common variant of the PCSK2 gene on reduced insulin secretion
}

\author{
A. Jonsson • B. Isomaa • T. Tuomi • J. G. Eriksson • \\ L. Groop • V. Lyssenko
}

Received: 27 July 2012 / Accepted: 21 August 2012 / Published online: 26 September 2012

(C) Springer-Verlag 2012

\begin{abstract}
Aim/hypothesis Individuals at risk of developing type 2 diabetes show a progressive decline in insulin secretion and increased insulin resistance over time. However, inability of the beta cells to compensate for the increased insulin resistance represents a key defect leading to overt type 2 diabetes. The aims of the present study were to replicate the association between genetic variants of the PCSK 2 gene and insulin secretion, and to explore the effect on risk of type 2 diabetes. Methods Replication of PCSK2 variants against insulin secretion included 7,682 non-diabetic Scandinavian individuals. Insulin secretion was measured as the corrected insulin response or disposition index, i.e. insulin secretion adjusted
\end{abstract}

Electronic supplementary material The online version of this article (doi:10.1007/s00125-012-2728-5) contains peer-reviewed but unedited supplementary material, which is available to authorised users.

A. Jonsson • L. Groop • V. Lyssenko ( $₫)$

Department of Clinical Sciences, Diabetes and Endocrinology, Lund University, CRC, Skåne University Hospital,

20502 Malmö, Sweden

e-mail: Valeriya.Lyssenko@med.lu.se

B. Isomaa $\cdot$ T. Tuomi $\cdot$ J. G. Eriksson

Folkhalsan Research Centre,

Helsinki, Finland

B. Isomaa

Department of Social Services and Health Care,

Jakobstad, Finland

T. Tuomi

Department of Medicine, Helsinki University Central Hospital, Helsinki, Finland

J. G. Eriksson

National Public Health Institute,

Helsinki, Finland for the degree of insulin resistance. Risk of type 2 diabetes was studied in 28,287 Scandinavian individuals.

Results The C-allele of PCSK2 rs2208203 was associated with reduced insulin secretion measured as the corrected insulin response $\left(n=8,151 ; \beta=-0.112, p=1.3 \times 10^{-6}\right)$ as well as disposition index $\left(n=8,078, \beta=-0.128, p=1.6 \times 10^{-7}\right)$. The variant was also associated with lower fasting glucagon levels $(\beta=-0.084, p=0.005)$ in non-diabetic individuals with a fasting plasma glucose of over $5.5 \mathrm{mmol} / \mathrm{l}$. In human pancreatic islets, $P C S K 2$ expression correlated negatively with $\mathrm{HbA}_{1 \mathrm{c}}(n=133, r=-0.196, p=0.038)$, and showed a tendency to be lower in hyperglycaemic $\left(\mathrm{HbA}_{1 \mathrm{c}} \geq 6.0 \%\right.$ or type 2 diabetes; $n=47, p=0.13$ ) than normoglycaemic

\section{J. G. Eriksson}

Department of General Practice and Primary Healthcare,

University of Helsinki,

Helsinki, Finland

\author{
J. G. Eriksson \\ Vasa Central Hospital, \\ Vasa, Finland
}

J. G. Eriksson

Unit of General Practice, Helsinki University Central Hospital, Helsinki, Finland

\footnotetext{
L. Groop

Finnish Institute of Molecular Medicine (FIMM),

Helsinki University,

Helsinki, Finland
} 
$\left(\mathrm{HbA}_{1 \mathrm{c}}>6.0 \% ; n=66\right)$ donors. The presence of the PCSK2 rs2208203 risk allele did not influence gene expression, nor did it show an apparent risk in terms of type 2 diabetes.

Conclusions/interpretation A variant of the PCSK2 gene was associated with reduced glucose-stimulated insulin secretion, but also with lower glucagon levels, which could potentially counteract the effects of decreased insulin secretion on the risk of type 2 diabetes.

Keywords Genetics · Insulin secretion · Type 2 diabetes

$\begin{array}{ll}\text { Abbreviations } \\ \text { BPS } & \text { Botnia Prospective Study } \\ \text { CIR } & \text { Corrected insulin response } \\ \text { DGI } & \text { Diabetes Genetics Initiative } \\ \text { DI } & \text { Disposition index } \\ \text { GWAS } & \text { Genome-wide association study } \\ \text { HBCS } & \text { Helsinki Birth Cohort Study } \\ \text { ISI } & \text { Insulin sensitivity index } \\ \text { MCC } & \text { Malmö Case-Control } \\ \text { MPP } & \text { Malmö Preventive Project } \\ \text { PC2 } & \text { Proprotein convertase subtilisin/kexin type 2 } \\ \text { PPP-Botnia } & \begin{array}{l}\text { Prevalence, Prediction and Prevention of } \\ \end{array} \\ \text { Diabetes Botnia study } \\ \text { SNP } & \text { Single nucleotide polymorphism }\end{array}$

\section{Introduction}

Individuals at risk of developing type 2 diabetes show a progressive decline in insulin secretion and increased insulin resistance over time. However, an inability of the beta cells to compensate for the degree of insulin resistance represents a key defect leading to overt type 2 diabetes. Several genome-wide association studies (GWASs) have identified a number of genetic loci resulting in impaired islet function and an increased risk of type 2 diabetes [1-5].

We previously identified an association between a common variant of the MTNRIB gene and reduced insulin secretion in the Diabetes Genetics Initiative (DGI) GWAS [6]. In addition, variants in the proprotein convertase subtilisin/kexin type 2 gene (PCSK2) were associated with reduced insulin secretion in the analysis of early insulin secretion in the DGI study, which included 999 non-diabetic individuals with information on glucose and insulin concentrations during an OGTT. The protein coded by this gene, PC2, is a proinsulin-processing enzyme that plays a key role in regulating insulin and glucagon biosynthesis. PCSK2 is expressed in the brain and the pancreatic islets. Variants of the PCSK1 and PCSK2 genes have previously been linked to type 2 diabetes and obesity [7-11]. The aims of the current study were to study the association between genetic variants of the PCSK2 gene and insulin secretion, and to explore the effect on risk of type 2 diabetes.

\section{Methods}

Study populations The DGI consisted of 1,464 individuals with type 2 diabetes and 1,467 euglycaemic controls from Finland and Sweden [2]; 999 of the control participants had data available on glucose and insulin levels at fasting, 30, and 120 min during OGTTs.

The Prevalence, Prediction and Prevention of Diabetes Botnia (PPP-Botnia) study is a population-based study from the Botnia region of western Finland. A total of 4,650 nondiabetic participants $(n=4,007$ who did not overlap with the DGI; fasting plasma glucose $<7.0 \mathrm{mmol} / 1$ and $2 \mathrm{~h}$ plasma glucose $<11.1 \mathrm{mmol} / \mathrm{l}$ ) were included in the current study [12].

The Botnia Prospective Study (BPS) started in 1990 on the west coast of Finland with the aim of identifying genes increasing susceptibility to type 2 diabetes in members of families with type 2 diabetes [13]. The prospective arm of the study included 2,770 non-diabetic family members and/ or their spouses (1,263 men and 1,507 women, mean age 45 years), 138 of whom developed type 2 diabetes during a 7.7-year [14] follow-up period [15, 16]. All participants were given information about exercise and a healthy diet, and were retested with an OGTT at 2-3-year intervals. Overall, 1,987 non-diabetic individuals with available longitudinal measurements of insulin and glucose concentrations during OGTTs were included in the analyses for insulin secretion, while 2,770 individuals were included in the analyses for type 2 diabetes.

The Helsinki Birth Cohort Study (HBCS) included 2,003 participants (928 men and 1,075 women) aged 56-70 years, who had been born as singletons between 1934 and 1944 . All participants underwent an OGTT [17]. The insulin secretion analyses included 1,688 individuals without diabetes.

The Malmö Case-Control (MCC) study consisted of 3,436 participants with diabetes from the local Scania Diabetes Registry [18] and 3,427 non-diabetic controls from the population-based Malmö Diet and Cancer study [19] in southern Sweden. All diabetic participants were of Scandinavian origin and had an age at onset of diabetes of over 35 years, a C-peptide level of $\geq 0.3 \mathrm{nmol} / 1$ and no glutamic acid decarboxylase (GAD) antibodies. Controls were also of Scandinavian origin and had a fasting blood glucose below $5.5 \mathrm{mmol} / \mathrm{l}$ and an $\mathrm{HbA}_{1 \mathrm{c}}$ of less than $6.0 \%$.

The Malmö Preventive Project (MPP) is a large population-based prospective study from the city of Malmö, Sweden, consisting of 33,346 Swedish individuals who participated in a health screening over the period 19741992 [20]. All participants underwent a physical examination, and each had blood drawn for measurements of fasting blood glucose and lipid concentration. A total of 16,651 participants, 3,089 of whom developed type 2 diabetes 
during a median 28.6-year follow-up period, were included in the current analyses [14]. A diagnosis of diabetes was confirmed from patient records or based on a fasting plasma glucose concentration greater than $7.0 \mathrm{mmol} / 1$.

Human pancreatic islets from 128 human cadaver donors were provided by the Nordic network for clinical islet transplantation. The experimental protocol for isolation of the islets was approved by the ethics committee of Uppsala University and performed in accordance with local institutional and Swedish national regulations.

Measurements Blood samples were drawn at -10, 0, 30, 60 and $120 \mathrm{~min}$ during the OGTT both at baseline and at follow-up for the BPS cohort, and at 0, 30 and $120 \mathrm{~min}$ for PPP-Botnia. Proinsulin was measured in pmol/l using human proinsulin RIA (Linco Research, St Charles, MO, USA), serum glucagon in $\mathrm{pg} / \mathrm{ml}$ using radioimmunoassay (Millipore, St Charles, MO, USA). The insulin sensitivity index (ISI) from the OGTT was calculated as $10,000 / \sqrt{ }$ (fasting plasma glucose $\times$ fasting plasma insulin $\times$ mean OGTT $T_{\text {glucose }} \times$ mean $O G T T_{\text {insulin }}$ ) [21]. Beta cell function was assessed as the corrected insulin response (CIR) during the OGTT $(C I R=100 \times$ insulin at $30 \mathrm{~min} /$ [glucose at $30 \mathrm{~min} \times($ glucose $30 \mathrm{~min}-3.89)]$. $)$ [22] or as the disposition index (DI), i.e. insulin secretion adjusted for insulin sensitivity $(D I=C I R \times I S I)$.

Genotyping Genotyping was performed using an Affymetrix $500 \mathrm{~K}$ chip (Affymetrix, Santa Clara, CA, USA) for the DGI cohort, with matrix-assisted laser desorption-ionisation time-of-flight mass spectrometry on a MassARRAY platform (Sequenom, San Diego, CA, USA) for the PPPBotnia cohort, and by an allelic discrimination method with a TaqMan assay on an ABI 7900 platform (Applied Biosystems, Foster City, CA, USA) for the remaining cohorts. We obtained an average genotyping success rate of $95.9 \%$, and the average concordance rate, based on $2,814(8.5 \%)$ duplicate comparisons, was 99.5\%. Hardy-Weinberg equilibrium was fulfilled in all populations studied $(p>0.05)$.

Glucose-stimulated insulin secretion and gene expression in human pancreatic islets Total RNA was isolated using an AllPrep DNA/RNA Mini Kit (Qiagen, Hilden, Germany). The quality and concentration of the RNA were measured using an Agilent 2100 bioanalyser (Agilent, Santa Clara, CA, USA) and Nanodrop ND-1000 equipment (Nanodrop, Wilmington, DE, USA), respectively. Glucose-stimulated insulin secretion was performed in high $(16.7 \mathrm{mmol} / \mathrm{l})$ and low $(1.0 \mathrm{mmol} / \mathrm{l})$ concentrations of glucose in the medium as previously described [23]. Glucose-stimulated insulin secretion was expressed as the fold change in insulin release from the islets by comparing release at $16.7 \mathrm{mmol} / \mathrm{l}$ with release using the $1.0 \mathrm{mmol} / \mathrm{l}$ glucose medium. The microarrays were performed following the Affymetrix standard protocol as previously described [23].

Statistical analysis Variables in Table 1 are presented as mean $\pm \mathrm{SD}$, and if not normally distributed as median (interquartile range). The risk of developing type 2 diabetes is expressed as the OR using logistic regression analyses adjusted for age, sex and BMI. Genotype-phenotype correlations were studied using linear regression analyses adjusted for age, sex and BMI. The BPS was analysed using corrected residuals in PLINK software (http://pngu.mgh.harvard.edu/ $\sim$ purcell/plink/) [24] with the family-based association tests for quantitative traits (QFAM-total) method with adaptive permutation to correct for family structure. Non-normally distributed variables (CIR, DI, ISI and proinsulin and glucagon levels) were logarithmically (natural log) transformed for analysis. For analysis of data from human pancreatic islets, a non-parametric $t$ test was used to assess the association between genotype and phenotype (expression and insulin secretion), and Spearman's rank correlation test was used for the association between PCSK2 mRNA and $\mathrm{HbA}_{1 \mathrm{c}}$ levels. All statistical analyses were performed using IBM SPSS Statistics version 19.0 (IBM, Armonk, NY, USA) and PLINK version 1.07. Meta-analyses were performed with the Metan analytical tool package implemented in STATA SE v. 10.1 (StataCorp, College Station, TX, USA) using fixed-effect models with the inverse variance method. Two-sided $p$ values of less than 0.05 were considered statistically significant.

\section{Results}

Effect of genetic variants in the PCSK2 gene on insulin secretion In the DGI GWAS [2, 6], we identified a PCSK2 locus as being consistently associated with insulin secretion in both non-diabetic and diet-treated diabetic individuals $(p<0.01)$ (see electronic supplementary material [ESM] Table 1) and selected five single nucleotide polymorphisms (SNPs) for follow-up validation in the non-diabetic participants from the PPP-Botnia study (ESM Table 2). We found two SNPs, rs2208203 and rs6034804 $\left(D=0.85, \mathrm{r}^{2}=0.62\right)$, in the PCSK2 gene that were associated $\left(p<1 \times 10^{-4}\right)$ with insulin secretion in this replication study of approximately 2,800 participants and remained significant after Bonferroni correction.

Next, we examined whether the association between our strongest replication signal of PCSK2 rs2208203 and reduced insulin secretion could be replicated in additional cohorts (Tables 1 and 2). In line with the effect of the variant of PCSK2 in the DGI and PPP-Botnia study, C-allele carriers of rs2208203 showed decreased insulin secretion in the HBCS study (CIR $\beta=-0.103$ [SE 0.043], $p=0.018$; DI $\beta=-0.135$ [SE 0.045 ], $p=0.0025$ ). The meta-analysis of all studies, totalling more than 8,100 individuals, showed that 


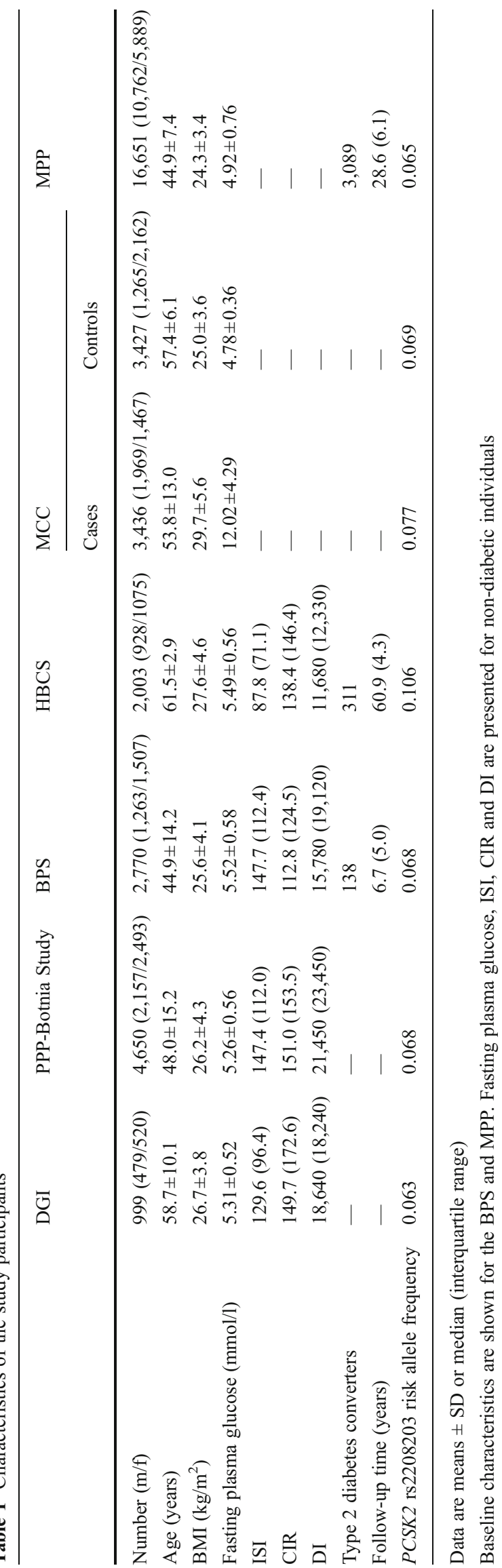


Table 2 Effects of PCSK2 rs2208203 on insulin secretion and sensitivity in non-diabetic individuals from the populations studied

\begin{tabular}{|c|c|c|c|c|}
\hline & $n$ & $\beta$ & SE & $p$ value \\
\hline \multicolumn{5}{|l|}{ DGI study } \\
\hline Insulin secretion (CIR) & 999 & -0.191 & 0.099 & 0.060 \\
\hline DI & 999 & -0.260 & 0.084 & 0.0023 \\
\hline ISI & 999 & 0.006 & 0.098 & 0.95 \\
\hline \multicolumn{5}{|l|}{ PPP-Botnia study } \\
\hline Insulin secretion (CIR) & 3,711 & -0.128 & 0.035 & $2.2 \times 10^{-4}$ \\
\hline DI & 3,641 & -0.108 & 0.037 & 0.0032 \\
\hline ISI & 3,655 & 0.014 & 0.023 & 0.55 \\
\hline \multicolumn{5}{|l|}{ BPS } \\
\hline \multicolumn{5}{|l|}{ Baseline } \\
\hline Insulin secretion (CIR) & 1,886 & -0.071 & 0.049 & 0.19 \\
\hline DI & 1,886 & -0.096 & 0.051 & 0.10 \\
\hline ISI & 1,886 & -0.025 & 0.032 & 0.49 \\
\hline \multicolumn{5}{|l|}{ Follow-up } \\
\hline Insulin secretion (CIR) & 1,886 & 0.011 & 0.050 & 0.84 \\
\hline DI & 1,886 & -0.025 & 0.055 & 0.69 \\
\hline ISI & 1,886 & -0.037 & 0.035 & 0.35 \\
\hline \multicolumn{5}{|l|}{ HBCS } \\
\hline Insulin secretion (CIR) & 1,559 & -0.103 & 0.043 & 0.018 \\
\hline DI & 1,556 & -0.135 & 0.045 & 0.0025 \\
\hline ISI & 1,558 & -0.031 & 0.031 & 0.32 \\
\hline \multicolumn{5}{|l|}{ Meta-analyses } \\
\hline Insulin secretion (CIR) & 8,151 & -0.112 & 0.023 & $1.3 \times 10^{-6}$ \\
\hline DI & 8,078 & -0.125 & 0.024 & $1.6 \times 10^{-7}$ \\
\hline ISI & 8,094 & -0.007 & 0.016 & 0.65 \\
\hline
\end{tabular}

$\beta$ and SE obtained from linear regression analysis adjusted for age, sex and BMI. The effect size is presented for each C-allele (additive model). Meta-analyses included the DGI study, PPP-Botnia study (participants not overlapping with the DGI study), HBCS and BPS at baseline

the $\mathrm{C}$-allele was associated with an $11 \%$ reduction in CIR $\left(p=1.3 \times 10^{-6}\right)$ and a $13 \%$ reduction in DI $\left(p=1.6 \times 10^{-7}\right)$ (Fig. 1). Furthermore, we observed that carriers of the risk C-allele had reduced fasting glucagon levels $(\beta=-0.084$ [SE
0.030], $p=0.0048$ ) in the hyperglycaemic (fasting plasma glucose $>5.5 \mathrm{mmol} / \mathrm{l}$ ) participants from the PPP-Botnia study (ESM Table 3). The SNP rs2208203 did not seem to influence proinsulin concentrations, nor did the variant have a significant effect on insulin sensitivity or BMI.

Effect of genetic variations in the PCSK2 gene on risk of type 2 diabetes Having observed an effect of the $\mathrm{C}$-allele of rs2208203 in PCSK2 in terms of reducing insulin secretion, we next asked whether this impairment of insulin secretion was translated into an increased risk of type 2 diabetes. In the MCC, the C-allele of rs2208203 was only weakly associated with risk of type 2 diabetes (OR 1.14, 95\% CI [0.98, 1.34], $p=$ 0.086). However, we were not able to validate the association of SNP rs2208203 with type 2 diabetes in our large prospective cohorts including 21,424 individuals followed for 27 years, of whom 3,538 developed diabetes (ESM Table 4).

Expression of PCSK2 and glucose-stimulated insulin secretion in human pancreatic islets We also analysed whether the level of glycaemia and/or the SNP influenced expression of the PCSK2 gene using microarray analysis of human pancreatic islets from 128 donors, 84 of whom also had information on glucose-stimulated insulin secretion. PCSK2 mRNA correlated negatively with $\mathrm{HbA}_{1 \mathrm{c}}$ levels $(r=-0.196$, $p=0.038, n=113$ ) (Fig. 2), and gene expression showed a tendency to be lower in hyperglycaemic $\left(\mathrm{HbA}_{1 \mathrm{c}} \geq 6.0 \%\right.$ or type 2 diabetes, $n=47$ ) compared with normoglycaemic $\left(\mathrm{HbA}_{1 \mathrm{c}}<6.0 \%, n=66\right)$ donors $(p=0.13)$. There was no significant correlation between PCSK2 expression and insulin release at high and low glucose concentrations, nor did the rs2208203 variant influence PCSK2 expression or insulin secretion (ESM Table 5).

\section{Discussion}

Here we report that a common variant of the PCSK2 gene is associated with reduced insulin secretion. PC2, the protein
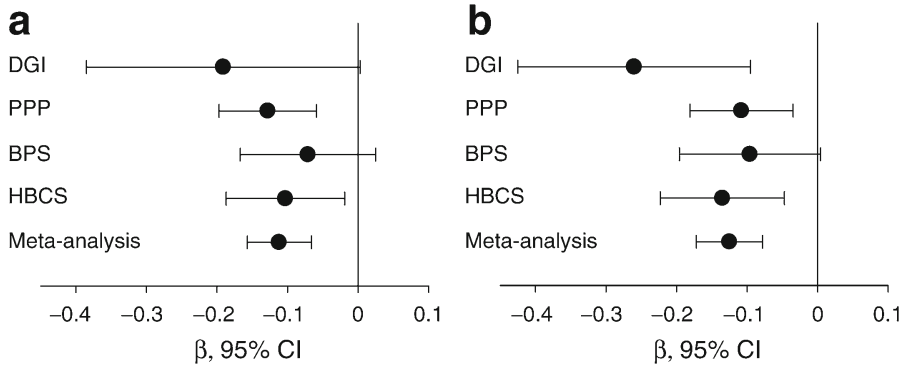

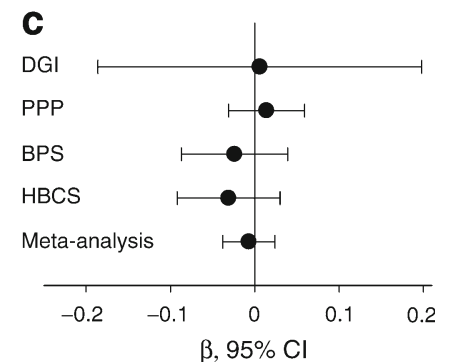

the $\mathrm{C}$-allele was associated with a reduced DI. Meta-analysis: $\beta=$ -0.125 (SE 0.024), $p=1.6 \times 10^{-7}$. (c) Insulin sensitivity; the C-allele did not show any significant effect on insulin sensitivity. Metaanalysis: $\beta=-0.007$ (SE 0.016), $p=0.65$
Fig. 1 Meta-analyses of PCSK2 rs2208203 for insulin secretion and insulin sensitivity. (a) CIR; the C-allele was associated with reduced insulin secretion. Meta-analysis: $\beta=-0.112$ (SE 0.023), $p=1.3 \times 10^{-6}$. (b) DI, i.e. insulin secretion adjusted for degree of insulin sensitivity; 


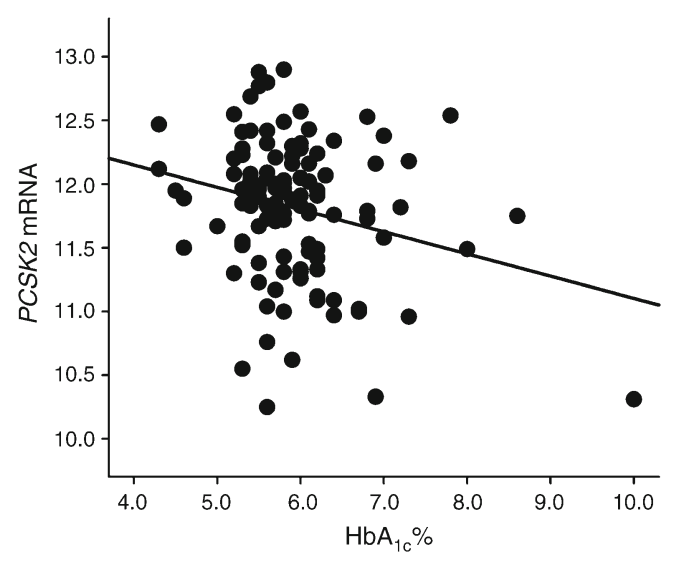

Fig. 2 Spearman correlation between $P C S K 2$ mRNA and $\mathrm{HbA}_{1 \mathrm{c}}$ in human islets. There was a negative correlation between PCSK2 expression and $\mathrm{HbA}_{1 \mathrm{c}}$ level $(r=-0.196, p=0.038, n=113)$. To convert values for $\mathrm{HbA}_{1 \mathrm{c}}$ in $\%$ into $\mathrm{mmol} / \mathrm{mol}$, subtract 2.15 and multiply by 10.929

encoded by PCSK2, is involved in the regulation of insulin and glucagon biosynthesis and is therefore a plausible candidate gene for the development of type 2 diabetes. Defects in the catalytic activity of prohormone enzymes would lead to abnormal hormone-processing. Although we were not able to measure PC2 activity in human islets, the lower PCSK2 expression observed in hyperglycaemic donors could indicate decreased concentrations of PC2 and in turn lead to decreased production of insulin and glucagon peptides in the islets. We cannot, however, exclude the effects on expression being secondary to a long-term increase in glucose levels as no correlation was seen between PCSK2 expression and insulin secretion in vitro. Notably, the PCSK2 variants were strongly associated with reduced early glucose-stimulated insulin secretion at 30 min during the OGTTs. This is in agreement with studies using MIN6 cells that demonstrate the rapid effects of glucose, occurring after a 20-min lag period and reaching maximum at $60 \mathrm{~min}$, on the regulation of both $\mathrm{PC} 2$ and proinsulin biosynthesis [25].

Given that PCSK2 variants affected neither proinsulin nor C-peptide levels, this would suggest primary effects on insulin synthesis. Pcsk2 knockout mice show decreased growth, reduced adiposity and protection from dietary fatinduced body weight gain. In addition, they exhibit fasting hypoglycaemia, reduced blood glucose and reduced circulating glucagon during intraperitoneal glucose tolerance testing. Processing of proglucagon and proinsulin is severely impaired in these mice [26, 27]. Furthermore, supporting the notion of PC2 effects on proglucagon processing, we observed that the PCSK2 variant was associated with lower glucagon levels in hyperglycaemic individuals.

The PCSK2 gene is located on chromosome 20p11.2 and consists of 12 exons with a protein coding region of
$257.6 \mathrm{kbp}$. Variants in the PCSK2 gene have previously been associated with type 2 diabetes in African [9], Japanese [10] and Chinese [11] individuals, but not, to our knowledge, European populations. Here we report an independent signal in intron 2 of PCSK2 in Scandinavian populations that was not correlated with previously reported variants in African (rs2021785), Japanese (microsatellite marker in intron 2) and Chinese (rs2021785) populations. Of note, none of the previously reported variants showed an association with insulin secretion in the DGI [2]. In the present study, the variant rs2208203 was not associated with risk of type 2 diabetes in a total of 21,313 non-diabetic and 6,974 diabetic individuals. One potential reason for the lack of apparent risk of type 2 diabetes could be the concomitant effect of PCSK2 variant on lowering glucagon levels.

In conclusion, a variant the PCSK2 gene (rs2208203) was associated with reduced glucose-stimulated insulin secretion, but also with lower glucagon levels that potentially counteracted the effects of decreased insulin secretion on risk of type 2 diabetes. These findings, together with several earlier observations, support an involvement of the PCSK2 gene in the pathogenesis of type 2 diabetes.

Acknowledgements Human islets were provided by the Nordic network for clinical islets transplantation courtesy of O. Korsgren, Uppsala, Sweden, and the expression studies were performed by the Human Tissue Laboratory at Lund University Diabetes Centre (LUDC). We thank the patients for their participation and the Botnia Study Group for clinical study of the patients.

Funding Studies at LUDC, Malmö were supported by grants from the Swedish Research Council, including a Linné grant (No. 349-20086589), a strategic research area grant Exodiab (2009-1039), the Heart and Lung Foundation, the Swedish Diabetes Research Society, the European Community's Seventh Framework Programme (grant CEED3, 223211), the Diabetes Programme at the Lund University, the Påhlsson Foundation, the Crafoord Foundation, the Knut and Alice Wallenberg Foundation, Novo Nordisk Foundation and the European Foundation for the Study of Diabetes. The Botnia studies were supported by grants from the Sigrid Juselius Foundation, the Folkhälsan Research Foundation, the Finnish Diabetes Research Society, the Signe and Ane Gyllenberg Foundation, the Swedish Cultural Foundation in Finland, the Ollqvist Foundation, Ministry of Education of Finland, the Foundation for Life and Health in Finland, Jakobstad Hospital, the Medical Society of Finland, the Närpes Research Foundation and the Vasa and Närpes Health centres.

Duality of interest The authors declare that there is no duality of interest associated with this manuscript.

Contribution statement AJ designed the study, researched and interpreted the data and wrote the manuscript. BI, TT and JGE collected and phenotyped the study populations. LG and VL designed and supervised the study, interpreted the data and contributed to discussion. All authors reviewed and approved the final version of the manuscript. 


\section{References}

1. Dupuis J, Langenberg C, Prokopenko I et al (2010) New genetic loci implicated in fasting glucose homeostasis and their impact on type 2 diabetes risk. Nat Genet 42:105-116

2. Saxena R, Voight BF, Lyssenko V et al (2007) Genome-wide association analysis identifies loci for type 2 diabetes and triglyceride levels. Science 316:1331-1336

3. Scott LJ, Mohlke KL, Bonnycastle LL et al (2007) A genome-wide association study of type 2 diabetes in Finns detects multiple susceptibility variants. Science 316:1341-1345

4. Voight BF, Scott LJ, Steinthorsdottir V et al (2010) Twelve type 2 diabetes susceptibility loci identified through large-scale association analysis. Nat Genet 42:579-589

5. Zeggini E, Weedon MN, Lindgren CM et al (2007) Replication of genome-wide association signals in UK samples reveals risk loci for type 2 diabetes. Science 316:1336-1341

6. Lyssenko V, Nagorny CL, Erdos MR et al (2009) Common variant in MTNR1B associated with increased risk of type 2 diabetes and impaired early insulin secretion. Nat Genet 41:82-88

7. Benzinou M, Creemers JW, Choquet H et al (2008) Common nonsynonymous variants in PCSK 1 confer risk of obesity. Nat Genet 40:943-945

8. Heni M, Haupt A, Schafer SA et al (2010) Association of obesity risk SNPs in PCSK1 with insulin sensitivity and proinsulin conversion. BMC Med Genet 11:86

9. Leak TS, Keene KL, Langefeld CD et al (2007) Association of the proprotein convertase subtilisin/kexin-type 2 (PCSK2) gene with type 2 diabetes in an African American population. Mol Genet Metab 92:145-150

10. Yoshida H, Ohagi S, Sanke T, Furuta H, Furuta M, Nanjo K (1995) Association of the prohormone convertase 2 gene (PCSK2) on chromosome 20 with NIDDM in Japanese subjects. Diabetes 44:389-393

11. Zheng X, Ren W, Zhang S et al (2012) Association of type 2 diabetes susceptibility genes (TCF7L2, SLC30A8, PCSK1 and PCSK2) and proinsulin conversion in a Chinese population. Mol Biol Rep 39:17-23

12. Isomaa B, Forsen B, Lahti K et al (2010) A family history of diabetes is associated with reduced physical fitness in the Prevalence, Prediction and Prevention of Diabetes (PPP)-Botnia study. Diabetologia 53:1709-1713

13. Groop L, Forsblom C, Lehtovirta M et al (1996) Metabolic consequences of a family history of NIDDM (the Botnia study): evidence for sex-specific parental effects. Diabetes 45:1585-1593
14. Lyssenko V, Jonsson A, Almgren P et al (2008) Clinical risk factors, DNA variants, and the development of type 2 diabetes. N Engl J Med 359:2220-2232

15. Lyssenko V, Almgren P, Anevski D et al (2005) Genetic prediction of future type 2 diabetes. PLoS Med 2:e345

16. Lyssenko V, Almgren P, Anevski D et al (2005) Predictors of and longitudinal changes in insulin sensitivity and secretion preceding onset of type 2 diabetes. Diabetes 54:166-174

17. Eriksson JG, Osmond C, Kajantie E, Forsen TJ, Barker DJ (2006) Patterns of growth among children who later develop type 2 diabetes or its risk factors. Diabetologia 49:2853-2858

18. Lindholm E, Agardh E, Tuomi T, Groop L, Agardh CD (2001) Classifying diabetes according to the new WHO clinical stages. Eur J Epidemiol 17:983-989

19. Hedblad B, Nilsson P, Engstrom G, Berglund G, Janzon L (2002) Insulin resistance in non-diabetic subjects is associated with increased incidence of myocardial infarction and death. Diabet Med 19:470-475

20. Berglund G, Nilsson P, Eriksson KF et al (2000) Long-term outcome of the Malmo Preventive Project: mortality and cardiovascular morbidity. J Intern Med 247:19-29

21. Matsuda M, DeFronzo RA (1999) Insulin sensitivity indices obtained from oral glucose tolerance testing: comparison with the euglycemic insulin clamp. Diabetes Care 22:1462-1470

22. Hanson RL, Pratley RE, Bogardus C et al (2000) Evaluation of simple indices of insulin sensitivity and insulin secretion for use in epidemiologic studies. Am J Epidemiol 151:190-198

23. Jonsson A, Isomaa B, Tuomi T et al (2009) A variant in the KCNQ1 gene predicts future type 2 diabetes and mediates impaired insulin secretion. Diabetes 58:2409-2413

24. Purcell S, Neale B, Todd-Brown K et al (2007) PLINK: a toolset for whole-genome association and population-based linkage analyses. Am J Hum Genet 81:559-575

25. Skelly RH, Schuppin GT, Ishihara H, Oka Y, Rhodes CJ (1996) Glucose-regulated translational control of proinsulin biosynthesis with that of the proinsulin endopeptidases PC2 and PC3 in the insulin-producing MIN6 cell line. Diabetes 45:3743

26. Anini Y, Mayne J, Gagnon J et al (2010) Genetic deficiency for proprotein convertase subtilisin/kexin type 2 in mice is associated with decreased adiposity and protection from dietary fat-induced body weight gain. Int J Obes (Lond) 34:15991607

27. Furuta M, Yano H, Zhou A et al (1997) Defective prohormone processing and altered pancreatic islet morphology in mice lacking active SPC2. Proc Natl Acad Sci U S A 94:6646-6651 